\title{
A comment on Suzuki's model for composite vector mesons
}

\author{
Andrew Cohen, Howard Georgi, and Elizabeth H. Simmons \\ Lyman Laboratory of Physics, Harvard University, Cambridge, Massachusetts 02138
}

(Received 25 January 1988)

\begin{abstract}
We show that Suzuki's model of composite vector bosons is consistent at the level of the effective low-energy theory. We generalize it to include couplings to quarks and leptons and discuss its phenomenology. We find observable corrections to the $W$ mass.
\end{abstract}

\section{INTRODUCTION}

An interesting heresy has recently been introduced by Suzuki. ${ }^{1}$ Suzuki suggests that the observed $W$ and $Z$ vector bosons are not gauge bosons at all, but are actually massive composites of fermionic constituents in a theory possessing only a global $\mathrm{SU}(2)$ symmetry, which nevertheless satisfies the usual relations of a spontaneously broken gauge theory to a high degree of accuracy. In this paper we explore the consistency of this notion. While we are not able to prove that the idea makes sense by constructing explicitly a renormalizable theory that implements this mechanism, we can and do construct selfconsistent effective field theories that realize it and show how to compute corrections to the usual gauge-theory relations.

Suzuki discusses a nonrenormalizable interaction involving $N$ sets of fermions, each set transforming as the fundamental representation of an $\mathrm{SU}(m)$ group. He assumes that the interactions of these fermions are described by an effective Lagrangian of the form

$$
\mathcal{L}=\sum_{j} \bar{\Upsilon}_{j}\left(i \gamma_{\mu} \partial^{\mu}-M\right) \Upsilon_{j}-\frac{1}{f^{2}} J_{a}^{\mu} J_{\mu a},
$$

where $J_{a}^{\mu}=\Sigma_{j} \bar{\Upsilon}_{j} \gamma^{\mu} T^{a} \Upsilon_{j}$ and $T^{a}$ is a generator of $\mathrm{SU}(m)$. He analyzes this theory in the $N \rightarrow \infty$ limit and argues that it produces a set of composite vector bosons with self-couplings identical to those of $\mathrm{SU}(\mathrm{m})$ gauge bosons with a gauge coupling of order $4 \pi / \sqrt{N}$ and mass of order $4 \pi f / \sqrt{N}$.

This imaginative idea immediately suggests at least three questions. Does the nonrenormalizable interaction (1.1) make any sense at all? How would we incorporate couplings of the composite vector bosons to the quarks and leptons? Does the resulting theory look anything like what we see in the couplings of quarks and leptons to the $W$ and $Z$ ? It is these questions that we discuss in this note.

\section{A PECULIAR LARGE- $\boldsymbol{N}$ LIMIT}

We begin by considering $N$ sets of fermions, each set transforming as the fundamental representation of an $\mathrm{SU}(m)$ group. We assume that the interactions of these fermions are described by an effective Lagrangian whose higher-dimension operators are characterized by a scale $\Lambda \equiv 4 \pi f$ :

$$
\mathcal{L}=\bar{\Upsilon}\left(i \gamma_{\mu} \partial^{\mu}-M\right) \Upsilon-\frac{1}{N^{\alpha} f^{2}} J_{a}^{\mu} J_{\mu a}+\cdots,
$$

where we have suppressed both $\mathrm{SU}(m)$ and $\mathrm{SU}(N)$ indices. The dots represent all other operators invariant under $\mathrm{SU}(m) \times \mathrm{SU}(N)$. Notice that these include several other four-fermion operators. The absence of these other four-fermion interactions in (1.1) is one of the peculiar things about Suzuki's model that we would eventually like to understand. We have also included a conventional factor of $N^{-\alpha}$ in the coefficient of the interaction term, where $\alpha$ is a parameter yet to be determined. The $N$ dependence of the operators in (2.1) will be chosen to allow the construction of scattering amplitudes perturbatively in inverse powers of $N$. The usual large- $N$ perturbation theory is obtained by taking $\alpha=1$. Suzuki's heresy is obtained by taking $\alpha=0$. Since we have not specified the dynamics which give rise to this effective Lagrangian we will leave $\alpha$ arbitrary, subject only to consistency of the low-energy theory.

In developing a perturbation theory in powers of $1 / N$, our first thought is that $\alpha=1$ is required to prevent the appearance of terms which grow like arbitrarily large positive powers of $N$. For example, the graph with $b$ bubbles of Fig. 1 has an $N$ dependence:

$$
\frac{N^{b(1-\alpha)}}{N^{\alpha}} \text {. }
$$

Clearly this grows unless $\alpha \geq 1$. However this large- $N$ behavior is not enough to exclude a perturbative expansion with $\alpha<1$. In fact these bubble diagrams form a geometric series which can be summed to give an effective interaction

$$
J_{a}^{\mu}\left(g_{\mu \nu}-\frac{q_{\mu} q_{v}}{q^{2}}\right) \frac{1}{N^{\alpha} f^{2}-N \Pi\left(q^{2}\right)} J_{a}^{v}+\frac{1}{N^{\alpha} f^{2}} J_{a}^{\mu} \frac{q_{\mu} q_{v}}{q^{2}} J_{a}^{v},
$$

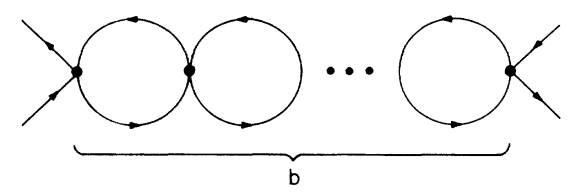

FIG. 1. Four-fermion interaction mediated by $b$ fermion loops. 


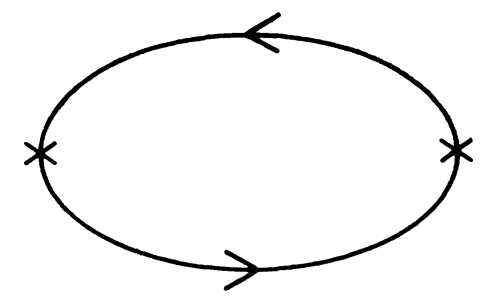

FIG. 2. Vacuum polarization $\Pi\left(q^{2}\right)$ due to a single fermion.

where $\Pi\left(q^{2}\right)$ is given by the graph of Fig. 2 .

If we ignore the second term, this interaction has an $N$ dependence which has been improved sufficiently to give rise to a $1 / N$ expansion that has no diagrams which grow faster than $1 / N^{\alpha}$. Naively we would have a good $1 / N$ perturbation theory. To examine the perturbation theory we must consider in detail the nature of $\Pi\left(q^{2}\right)$.

Before considering the above model we will find it instructive to examine a scalar-scalar interaction instead of a vector-vector one, i.e., the Gross-Neveu model (in four dimensions). Letting $\phi \equiv \bar{\Upsilon}(x) \Upsilon(x)$, the sum of graphs in Fig. 1 is

$$
\phi(q)\left[N^{\alpha} f^{2}-N G\left(q^{2}\right)\right]^{-1} \phi(-q),
$$

where $G\left(q^{2}\right)$ is given by the graph of Fig. 2 with $\phi$ insertions instead of current insertions. In four dimensions $G\left(q^{2}\right)$ is quadratically divergent and requires two subtractions to produce a finite result. The subtraction to remove the divergence of $G(0)$ corresponds to a renormalization of $N^{\alpha} f^{2}$, the coefficient of the interaction term. But this renormalization is of order $N$, which requires the renormalized interaction at zero momentum to have a coefficient of order $1 / N$, just the usual $1 / N$ counting. Summing up the bubbles has not really improved matters; it has just shown us that the only perturbative expansion in $1 / N$ that makes sense is the usual one with $\alpha=1$. If we take $\alpha<1$ the renormalized theory will have an $N$ dependence given by the $\alpha=1$ theory anyway.

Suzuki's observation is that the situation for the vector-vector theory is somewhat different. Although the graph is superficially quadratically divergent, it only requires a single subtraction to obtain a finite result. In fact the global $\mathrm{SU}(m)$ invariance of the theory ensures that $\Pi(0)=0$, and only the $q^{2}$ piece requires a renormalization. ${ }^{2}$ The coefficient of the interaction at zero momentum is therefore not renormalized by these graphs; only operators with extra powers of momenta receive corrections. Thus, in contrast with scalar theory the expansion in powers of $1 / N$ for this theory may be quite different for $\alpha<1$ then the traditional $\alpha=1$ case. For the remainder of our investigation we will take $\alpha=0$.

\section{AN AUXILIARY FIELD}

The perturbation theory will be improved through the use of the effective interaction in (2.3). In order to make the counting of powers of $N$ clear, we will replace the nonlocal interaction of (2.3) with the exchange of a mas- sive vector particle; that is, we will consider the Lagrangian

$$
\begin{aligned}
\mathcal{L}= & -\frac{1}{4} F_{a}^{\mu v} F_{a \mu \nu}+\bar{\Upsilon}\left(i \gamma_{\mu} \partial^{\mu}-M\right) \Upsilon-g J_{a \mu} A_{a}^{\mu} \\
& +\frac{m^{2}}{2} A_{a}^{\mu} A_{a \mu}+\cdots .
\end{aligned}
$$

When we integrate out the $A$ field this Lagrangian reduces to the Lagrangian of (2.3) if we make the identification

$$
g^{2} \simeq \frac{1}{\Pi^{\prime}(0) N}, \quad m^{2} \simeq \frac{f^{2}}{\Pi^{\prime}(0) N} .
$$

Note that we have done something drastic to the higher-dimension operators. If we had expanded (2.3) in powers of momenta, we would have said that operators with many derivatives also had many powers of $N$ - the expansion in $q^{2}$ forces us to expand the geometric series. But the $N$ counting of (3.1) does not suffer from this flaw; indeed we constructed this theory precisely in order to improve the $N$ counting. The reason for this apparent change is of course the appearance of the pole in (2.3). In the theory we started with, (2.1), we are not surprised by the appearance of a composite vector boson, because the strong interaction wants to bind fermions into composite vector states. However the appearance of a vector particle whose mass is much lighter than the scale of the interaction, $(4 \pi f)$, is something of a surprise. Indeed we might not believe it - in the original theory the higherdimension operators might grow with $N$ and move the pole to much higher energies. However we will show in Sec. IV that it is consistent to keep the composite vector light, with appropriate coefficients for the higherdimension operators; that is our investigation will provide necessary conditions for the appearance of a light composite vector boson. That is why we have treated the higher-dimension operators so cavalierly. We assume that the operators in the theory involving the $A$ field, (3.1), have coefficients which lead to a well-defined effective field theory, and that these coefficients come from operators in the theory of (2.1) with appropriate coefficients.

The field $A_{a}^{\mu}$ is Suzuki's composite vector boson. In this view, the gauge invariance of the self-couplings of $A$ is obvious. The Lagrangian $(3.1)$ has an $\mathrm{SU}(m)$ gauge symmetry that is broken only by the mass term for the $A$ field and (possibly) by the terms that we have not written explicitly.

Producing a light composite state is important because we are only working in an effective low-momentum field theory. If the pole appears at a scale which is of the order of the scale at which our effective theory breaks down, we will not believe it. (This is the case for the scalar-scalar theory, where this state is just the Landau ghost.) However in Suzuki's theory, the mass of the state is

$$
m^{2} \simeq g^{2} f^{2},
$$

where $g \simeq 1 / N$. Thus $m$ is much less than the scale $\Lambda=4 \pi f$ where the theory ceases to be valid. 


\section{BUILDING THE EFFECTIVE THEORY}

Our first goal is to understand the Lagrangian of (1.1) by constructing an effective low-energy theory containing the interaction in (1.1), but in which the higher dimension operators are controlled by a consistent power counting in both $1 / \Lambda$ and $1 / N$ (Ref. 3 ). We have already taken the first step, by introducing the auxiliary field in (3.1). Note that when we introduced the $A$ field to eliminate the four-fermion interaction in (1.1), we were forced to include a kinetic energy term for the $A$ field. Had we not done so, such a term would have been produced by renormalization anyway. However, we have not yet reached our goal. The remaining problem stems from the mass term for the $A$ field, which behaves like $1 / \sqrt{N}$. The limit in which the $A$ mass goes to zero like $1 / \sqrt{N}$ is a dangerous one because of the longitudinal part of the $A$ propagator, which blows up like $N$. This means that the longitudinal part of the massive vector particle has an $N$ counting that is worse than the transverse part by a factor of $N$. This is obvious by the form of (2.3) where we had previously neglected the longitudinal component. This is potentially harmful, because a graph with $L$ fermion loops will have a factor $N^{L}$ from the index loops and, if we saturate all vector interactions with the longitudinal piece of the vector field, no compensating powers of $1 / N$.

The large positive power of $N$ in this graph would force us to include operator coefficients proportional to large powers of $N$, destroying our ability to construct a good $1 / N$ expansion. Our only hope is that, just as we were able to sum up the potentially troublesome contributions in the simple bubble chain, we could sum more complicated terms to leave contributions proportional only to inverse powers of $N$. Although the existence of such a cancellation seems unlikely, in fact the global $\mathrm{SU}(m)$ symmetry of the theory ensures that just such a cancellation occurs.

In order to explain how this cancellation works, we will need to rewrite the theory in a way that makes the implications of the $\mathrm{SU}(m)$ symmetry manifest. The theory specified by the Lagrangian of (3.1) contains all possible operators constructed out of $\Upsilon, A$, and their derivatives invariant under the global $\mathrm{SU}(m)$ symmetry, where $A$ transforms as an adjoint.

The terms of smallest dimension that we have written down in (3.1), except for the mass term for $A$, are invariant under a local SU $(m)$ gauge symmetry where $A$ transforms as a conventional gauge field. This symmetry is of course a fake, because it is spoiled by higher dimension operators as well as by the mass term. Nevertheless, because we are interested in constructing an expansion at low momentum, it will prove convenient to write the theory as if it has the local symmetry.

To do this, we will introduce a spurion - a set of fields that transform under the symmetry so as to restore the invariance under the local group, but do not change the physics of the theory; that is, the functional integral over the spurions should leave us with the Lagrangian of (3.1). The fields we introduce will transform nonlinearly under the $\mathrm{SU}(m)$ :

$$
\Sigma \equiv e^{2 i \phi / f} \rightarrow U \Sigma,
$$

where $\phi$ is an adjoint of scalar fields. The $A$ field can then be interpreted as a gauge field for this local $\mathbf{S U}(m)$, and the fermions assumed to transform as $m s$. Then the Lagrangian can be rewritten

$$
\begin{aligned}
\mathcal{L}= & \bar{\Upsilon}\left(i \gamma_{\mu} D^{\mu}-M\right) \Upsilon-\frac{1}{4} F_{a}^{\mu \nu} F_{a \mu \nu} \\
& +\frac{f^{2}}{4} \operatorname{tr}\left(D^{\mu} \Sigma D_{\mu} \Sigma^{\dagger}\right)+\cdots,
\end{aligned}
$$

where $D^{\mu}=\partial^{\mu}+i g T_{a} A_{a}^{\mu}$.

This Lagrangian possesses a local $\mathrm{SU}(m)$ gauge symmetry, specified by taking $U$ in (4.1) to be an arbitrary local function of space-time, which allows us to perform the functional integration over $\phi$ by simply fixing the gauge $\Sigma=1$. Then $D^{\mu} \Sigma=i g T_{a} A_{a}^{\mu}$. The important observation is that every operator that is invariant under the global $\mathrm{SU}(\mathrm{m})$ symmetry can be rewritten in terms of auxiliary fields $\phi$ in such a way as to yield an operator invariant under a local $\mathrm{SU}(m)$ symmetry.

In the next section, we will generalize (4.2) slightly to allow for a nontrivial structure for the fermion mass matrix, $M$. For now, let us note that in the absence of $M$, (4.2) has an $\mathrm{SU}(N) \times \mathrm{SU}(N) \times \mathrm{U}(1)$ chiral symmetry [or for $m=2$, an $\mathrm{SU}(2 N)$ ]. The mass term explicitly breaks this chiral symmetry down to $\mathrm{SU}(N)$ [or $\mathrm{Sp}(2 N)$ ]. Thus we can keep track of the factors of $M$ in the higher dimension operators by classifying them according to their transformation properties under the explicitly broken chiral symmetry.

We are finally in a position to complete our powercounting argument. The Lagrangian of (4.2) is equivalent to (3.1) when we choose unitary gauge. It is also gauge invariant, and therefore equivalent to (3.1) in any gauge, for example, the Landau gauge. However, in the Landau gauge the power counting is very simple. The $A$ propagator has no longitudinal part, but the $\Sigma$ field involves propagating Goldstone bosons with nonrenormalizable interactions. It is only through the interactions of the $A$ field with the Goldstone bosons that the nonrenormalizable interactions enter into the fermion sector of the theory. Counting powers of $\Lambda$ and $f$ are the same as they are for a conventional effective theory - each derivative or factor of $M$ is suppressed by a power of $\Lambda$. In addition we assign to each operator a power of $1 / N$ for each power of $g^{2} / 16 \pi^{2}$ in the leading renormalization of the operator by gauge interactions. No such powers are required to produce self-couplings of the $A$ fields, but to produce a renormalization of any operator involving $k$ fermion bilinears (except the fermion kinetic energy term itself) requires at least $1 / N^{k+1}$.

For example, for the operator

$$
\operatorname{tr}\left(D^{\mu} \Sigma D_{\mu} \Sigma^{\dagger} D^{v} \Sigma D_{v} \Sigma^{\dagger}\right)
$$

we expect a coefficient $1 / 16 \pi^{2}$. A renormalization of this order arises from Goldstone-boson loops. Then there will be no renormalization of this operator larger than the coefficient of the operator itself, and conversely it will not renormalize other operators with coefficients larger than their tree-level values. The operator (4.3) produces small 


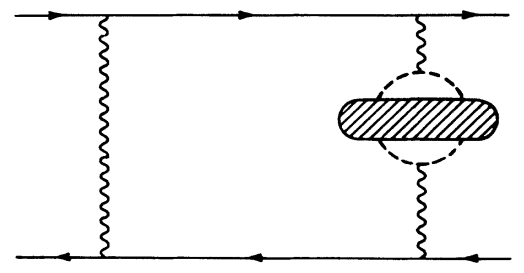

FIG. 3. Class of diagrams responsible for the leading renormalization of the four-fermion operator $\bar{\Upsilon} \gamma^{\mu} \Upsilon \bar{\Upsilon} \gamma_{\mu} \Upsilon$. Wavy lines represent composite gauge bosons $A^{\mu}$. Dashed lines represent Goldstone bosons $\Sigma$.

corrections to the self-couplings of the $A$ fields in unitary gauge, smaller than the terms in (4.2) by $1 / N$.

As another example, consider the operator

$$
\bar{\Upsilon} \gamma^{\mu} \Upsilon \bar{\Upsilon} \gamma_{\mu} \Upsilon \text {. }
$$

This is one of the four-fermion operators we discussed above that was not included in (1.1). We can now see that it is at least consistent to leave it out. The leading contribution to the renormalization of such a term comes from the class of diagrams shown in Fig. 3. These give a coefficient of order $1 /\left(N^{2} f^{2}\right)$.

One might well ask what we have gained from the above analysis. We believe that the existence of such an effective theory is a necessary condition for any sensible interpretation of the Lagrangian (1.1). In retrospect, it is not surprising that we have succeeded in producing such an effective theory, since the Suzuki model is designed to reproduce the low-energy physics of a spontaneously broken gauge symmetry which is certainly consistent. It is not clear, however, that the existence of a consistent effective theory is a sufficient condition for (1.1) to make sense. To really be sure, we should exhibit a renormalizable theory that produces (1.1) as a low-energy limit. This we have been unable to do, for reasons that we will discuss in our conclusions.

\section{COUPLINGS TO LIGHT FERMIONS}

So far our theory describes a set of composite vector bosons whose self-couplings at low energies are approximately those of the $W$ and $Z$ that would have come from a spontaneously broken gauge theory, along with a set of heavy fermions which interact with these vector bosons through approximate gauge couplings. In order to complete our theory we must include the ordinary quarks and leptons in a way which incorporates the successful features of a gauge theory. The obvious challenge in the coupling to the light fermions is to preserve the observed universality of the weak interactions. The appropriate couplings of the quarks and leptons to the composite $W$ and $Z$ will arise from a four-fermion coupling in the Lagrangian (1.1), which involves two heavy fermions and two ordinary quarks and leptons. In order to ensure the universality of the couplings, the coefficients of these four-fermion operators must be equal. The key is to regard the light fermion doublets as part of the $\Upsilon$ multiplet out of which the $W$ and $Z$ are built. The point, as antici- pated in the previous section, is that Suzuki's mechanism does not require an $\mathrm{SU}(N)$ symmetry of the constituents. Thus a mass term which splits the heavy fermions from the light quarks and leptons does not disturb the Suzuki mechanism. In fact, even terms that break the $\mathrm{SU}(m)$ symmetry are not a disaster, as long as they do not upset the peculiar large- $N$ limit discussed above.

In particular, let us specialize to $m=2$ and consider an $\Upsilon$ multiplet of left-handed fields of the form

$$
\Upsilon_{L} \equiv\left(\begin{array}{c}
\xi \\
i \tau_{2} \xi_{C} \\
\psi \\
L
\end{array}\right)_{L},
$$

where $\psi$ describes the three left-handed quark doublets, $L$ the three left-handed lepton doublets, and $\xi$ the $N$ Suzuki fermions. We have made use of the fact that the SU(2)doublet representation is equivalent to its complex conjugate to replace $\xi_{R}$ with the left-handed charge-conjugate field

$$
\xi_{C}=C \xi_{R}^{*},
$$

where $C$ is the charge-conjugation matrix. In addition, we need the right-handed fields $U_{R}, D_{R}$, and $l_{R}^{-}$to describe the SU(2)-singlet quark and lepton fields. Our generalization of the Suzuki Lagrangian has the form

$$
\begin{aligned}
\mathcal{L}= & \overline{\Upsilon_{L}}\left(i \gamma_{\mu} \partial^{\mu}-M\right) \Upsilon_{L}-\frac{1}{v^{2}} J_{a}^{\mu} J_{\mu a} \\
& +\overline{U_{R}} \gamma^{\mu} D_{\mu} U_{R}+\overline{D_{R}} \gamma^{\mu} D_{\mu} D_{R}+\overline{l_{R}} \gamma^{\mu} D_{\mu} l_{R} \\
& +\overline{U_{R}} M_{U} \Upsilon_{L}+\overline{D_{R}} M_{D} \Upsilon_{L}+\overline{l_{R}^{-}} M_{l} \Upsilon_{L}+\text { H.c. }+\cdots
\end{aligned}
$$

where

$$
\begin{aligned}
& M=i \tau_{2} C \gamma^{0}\left(\begin{array}{cccc}
0 & -M & 0 & 0 \\
M & 0 & 0 & 0 \\
0 & 0 & 0 & 0 \\
0 & 0 & 0 & 0
\end{array}\right), \\
& M_{U}=\left(\begin{array}{llll}
0 & 0 & M_{U} & 0
\end{array}\right) i u \tau_{2}, \\
& M_{D}=\left(\begin{array}{llll}
0 & 0 & M_{D} & 0
\end{array}\right) u, \\
& M_{l}=\left(\begin{array}{llll}
0 & 0 & 0 & \left.M_{l}\right) u
\end{array}\right.
\end{aligned}
$$

with $u=\left(\begin{array}{ll}0 & 1\end{array}\right)$ and $M_{U}, M_{D}$, and $M_{l}$ the up-quark, down-quark, and charged-lepton mass matrices. Here the covariant derivative incorporates $\mathrm{QCD}$ and electromagnetism, but of course, no gauge $\mathrm{SU}(2)$. In (5.3), we have replaced the arbitrary scale $f$ with $v \approx 250 \mathrm{GeV}$, to reproduce the usual strength of the weak interactions.

Equation (5.3) describes a theory with a global $\mathrm{SU}(2 N+12) \times \mathrm{SU}(21)$ symmetry explicitly broken by the relatively weak QCD and electromagnetic interactions and by the mass terms down to $\mathrm{SU}(N)$ and flavor $\mathrm{U}(1)$ 's. The mass terms are designed to give rise to the observed 
light-quark and lepton masses, while making the remaining fermions degenerate and heavy. In the powercounting arguments of the previous section, all these symmetry-breaking effects are small as long as the masses are of the order of the $W$ mass or smaller. The (now approximate) $\mathrm{SU}(2 N+12)$ symmetry is sufficient to guarantee universality of the weak interactions, or equivalently, that the coupling of the composite $W$ and $Z$ to the light fermions has the same structure as in the standard model with spontaneously broken $\mathrm{SU}(2)$ gauge invariance, up to small corrections.

\section{PHENOMENOLOGY OF COMPOSITE $W$ 's AND $Z$ 's}

The model that we have described in the preceding sections is nearly indistinguishable from that of a theory in which there is a spontaneously broken $\mathrm{SU}(2)$ gauge interaction at energies small compared to $1 \mathrm{TeV}$, but in which the fermions include not only the quarks and leptons, but $N$ Dirac SU(2) doublets. We will refer to these $N$ doublets as the Suzuki fermions. As we have seen, if these Suzuki fermions are much heavier than the $W$, then the explicit breaking of the $\mathrm{SU}(2 N+12)$ symmetry by the heavy-fermion mass terms will give rise to large terms in the effective Lagrangian that destroy the usual gauge theory relations. In particular the strength of the weak interactions and the masses of the $W$ and $Z$ will no longer be related by the standard-model formula.

On the other hand, the Suzuki fermions cannot be very light either, or we would have seen them. They cannot be much lighter than half $M_{W}$, or they would make an enormous contribution to the width of the $W$ and $Z$ which would have been observed at the CERN collider. Even if the $W$ and $Z$ cannot decay into physical pairs of Suzuki fermions, their coupling to virtual pairs of Suzuki fermions can give rise to measurable corrections to standard-model properties. It is this effect that we will discuss here.

One might naively expect this effect to rule out Suzuki fermions with a mass $M$ of order $M_{W}$ or less, because we would expect the couplings of the gauge bosons to the Suzuki fermions to produce contributions to the vectorboson form factors of order $p^{2} / M^{2}$. This in turn, would modify the position of the poles in the vector-boson propagators by factors of order $1+M_{W}^{2} / M^{2}$, completely invalidating the standard-model predictions for the vectorboson masses. However, as we will see, the form factor is much smaller than this estimate, because of the accidental appearance of a small number in the relevant Feynman diagram.

In order to investigate these corrections, we will look at the contribution of the Suzuki fermions to the vacuum polarization of the $W$ and $Z$ propagators. The Feynman diagram is logarithmically divergent. If we cut it off at a momentum of order $\Lambda$, the divergent part becomes

$$
\left(p^{2} g^{\mu \nu}-p^{\mu} p^{v}\right) \frac{N g^{2}}{12 \pi^{2}} \ln \left(\frac{\Lambda^{2}}{M^{2}}\right),
$$

where $g$ is the "observed" $\mathrm{SU}(2)$ gauge coupling, $\sqrt{4 \pi \alpha} / \sin \theta$. We expect $(6.1)$ to be of order 1 , since these fermion loops are responsible for generating the $W$ kinet- ic energy term in Suzuki's model. This argument is of course equivalent to the argument used in our identification of the gauge coupling in Sec. III (Ref. 4). Setting (6.1) equal to 1 with $\Lambda=1 \mathrm{TeV}$ and $M \approx M_{W}$ gives

$$
N \approx 60 \text {. }
$$

To compute the corrections to the standard-model parameters quantitatively, we need the finite, oncesubtracted part of the vacuum polarization that contributes to the $W$ form factor. In order to make the calculation as transparent as possible, we will perform the subtraction at zero momentum, which will make the physical meaning of the one-loop correction manifest. If we use low-energy weak interaction data to determine the parameters of the standard model, we are sensitive, in lowest order, to the $W$ propagator only for $p \approx 0$. The strength of the weak interactions thus determines only the $W$ mass term in the effective Lagrangian, which is not renormalized to leading order in $N$. The vacuumpolarization graph, subtracted at zero momentum, produces a correction to the kinetic term in the effective action, which vanishes at zero momentum. This will affect the position of the physical $W$ pole, without changing the strength of the weak interactions; the physical mass of the $W$ will not be where we expect it from the tree approximation. Because $N$ is large, this correction will be larger than other radiative corrections which are suppressed by powers of $1 / N$, so we can neglect them as well. Then to leading order, the $W$ mass is

$$
M_{W}^{2} \approx \frac{M_{W_{0}}^{2}}{1+N I\left(M_{W_{0}}^{2} / M^{2}\right)},
$$

where $M_{W_{0}}$ is the tree approximation to $M_{W}$ and $I(A)$ is the vacuum-polarization contribution:

$$
\begin{aligned}
I(A)=\frac{g^{2}}{2 \pi^{2}}\left[\frac{5}{18}+\frac{2}{3 A}-\right. & {\left[\frac{2 A-A^{2}+8}{3 A \sqrt{4 A-A^{2}}}\right] } \\
& \left.\times \arctan \left(\frac{A}{\sqrt{4 A-A^{2}}}\right]\right] .
\end{aligned}
$$

For small $A, I(A) \approx A / 30$. It is this factor of $\frac{1}{30}$ that makes the correction to $M_{W}$ phenomenologically consistent in spite of (6.2). In particular, for $N=60$ and $M=M_{W}$ (6.3) and (6.4) decrease $M_{W}$ by about $2 \%$. This is consistent with what we know today, but should be testable at CERN LEP and the Stanford Linear Collider.

Similar corrections can be derived for the $Z$ mass; however, these are more model dependent, because they depend on the average charge of the Suzuki fermions, which is otherwise not determined.

\section{CONCLUSIONS}

Suzuki's suggestion that the Lagrangian of Eq. (1.1) gives rise to composite vector bosons has lead us finally to 
the effective Lagrangian of Eq. (4.2). This effective theory has a consistent power counting and leads to an interesting phenomenology which differs from the standard model in a calculable way. Although (1.1) possesses only global symmetries and fundamental fermionic fields, the low-energy effective theory embodies all the successful features of a spontaneously broken gauge theory.

We have succeeded in interpreting the theory (1.1) by the construction of our effective Lagrangian, but our ultimate goal is to offer an explicit realization of the idea, in the form of a renormalizable theory that leads to (1.1) at long distances. This we have been unable to do. Our difficulty is not just a technical question, but one of principle, since the obstacle we have encountered in constructing such a theory is closely connected to the fundamental miracle that allowed the construction of the lowenergy effective Lagrangian in the first place. We were able to construct such a low-energy theory only because of the fact that the four-fermion interaction does not renormalize itself in leading order in $N$. This in turn allowed us to construct a peculiar large- $N$ limit that is con- sistent, yet leads to different physics from the conventional large- $N$ limit. However, in order to obtain the $N$ dependence indicated in the four-fermion interaction of (1.1), the renormalizable theory at high energy must also give rise to a peculiar large- $N$ counting. If the renormalizable theory at high energy does not contain some ana$\log$ of the Suzuki miracle, we have no hope of obtaining this peculiar large- $N$ limit. So far we have been unable to construct a theory which produces this abnormal set of counting rules.

\section{ACKNOWLEDGMENTS}

We thank V. Bhansali for helpful discussions. One of us (A.C.) thanks the Harvard Society of Fellows for financial support; another of us (E.H.S.) acknowledges that this material is based upon work supported by the $\mathrm{Na}$ tional Science Foundation Graduate Program. This work was supported in part by NSF Contracts Nos. PHY-8215249 and PHY-87-14654.
${ }^{1}$ M. Suzuki, Phys. Rev. D 37, 210 (1988).

${ }^{2}$ See, also, G. Parisi, Nucl. Phys. B100, 368 (1975).

${ }^{3}$ A. Manohar and H. Georgi, Nucl. Phys. B234, 189 (1984); H. Georgi and L. Randall, ibid. B276, 241 (1986).

${ }^{4} \mathrm{~A}$ renormalization of the $W$ propagator changes the coupling of the $W$ to the fermions. In order to give the correct value for the coupling constant, the kinetic term induced by the fermions must have a value consistent with this coupling; that is it must not require any further renormalization which would disturb the coupling. That is to say, the residue must be approximately one. This is, in fact, how we identified the effective gauge coupling in (3.2). 